\title{
System Level Design of the ITER Bolometer Port Plug Cameras ${ }^{a)}$
}

\author{
F. Penzel, ${ }^{1, b)}$ H. Meister, ${ }^{1}$ D. Hermann, ${ }^{1}$ A. Pataki, ${ }^{1}$ E. Walcz, ${ }^{2}$ G. Nadasi, ${ }^{2}$ Z. Szabo-Balint,${ }^{2}$ L. Tatar,${ }^{3}$ U. \\ Walach, ${ }^{4}$ L. C. Ingesson, ${ }^{4}$ R. Reichle, ${ }^{5}$ and J. Sanchez ${ }^{5}$ \\ ${ }^{1)}$ Max Planck Institute for Plasma Physics, Boltzmannstr. 2, 85748 Garching - Germany \\ ${ }^{2)}$ Wigner Research Centre for Physics, 29-33, Konkoly-Thege M. u. 1121, Budapest-Hungary \\ ${ }^{3)}$ Centre for Energy Research, Hungarian Academy of Sciences, 29-33, Konkoly-Thege M. u. 1121, \\ Budapest - Hungary \\ 4) Fusion for Energy, c/ Josep Pla n 2, Torres Diagonal Litoral B3, 08019 Barcelona - Spain \\ 5) ITER Organization, Route de Vinon-sur-Verdon, CS 90 046, 13067 St. Paul Lez Durance Cedex - France
}

(Dated: 8 June 2018)

The ITER bolometer diagnostic is planned to have 550 lines of sight (LOS) distributed all over the vessel. 240 channels are provided by cameras mounted in two Upper Ports and in one Equatorial Port. This paper describes the current status of the system level design of the port cameras and the solutions proposed on how to implement all required camera components while meeting a multitude of competing requirements. Sensor holders, support structures and different apertures depending on the camera type (pinhole or collimator), cable connectors, ceramic track plates and many mineral insulated cables have to be integrated within a restricted space envelope to guarantee functionality. The design of the internal electrical interfaces and the external mechanical mountings will be described as well. Using the example of an Upper Port camera with 60 LOS, the assembly of the camera components is explained and two currently discussed architecture options for the remote handling maintenance scheme in the hot cell are compared.

PACS numbers: 52.70.-m, 28.52.Lf, 29.40.n, 07.57.Kp

\section{INTRODUCTION AND REQUIREMENTS TO THE BOLOMETER DIAGNOSTIC}

ITER will be equipped with a thin-film metal resistor bolometer diagnostic ${ }^{1}$ to measure the total radiated power, part of the ITER energy balance of the plasma. The diagnostic measures in a broad spectral bandwidth and can provide a two-dimensional poloidal radiation distribution using tomography. 550 Bolometer lines of sights (LOS) are foreseen: 200 LOS from cameras mounted in 5 divertor cassettes, 110 LOS from cameras mounted on the vacuum vessel and 240 LOS from cameras mounted in two upper and one equatorial port ${ }^{2}$. Therefore, multiple sensors have to be integrated using a pinhole-type and/or collimator-type camera design to determine the required LOS orientation and characteristic. The defined performance requirements are $5 \mathrm{~cm}$ of lateral resolution resulting in LOS cones $<1^{\circ}$ at full width half maximum ${ }^{3}$, $1 \mathrm{~ms}$ time resolution and $10-20 \%$ of max. measurement uncertainty. There is no planned maintenance as the diagnostic is classified remote handling $(\mathrm{RH})$ class III. All components must be designed for ITER's lifetime and for the long pulse D-T operation load environment which results in high plasma and nuclear loads. Design driving are also the limited space and viewing access through the blankets, the attachment requirements imposed by the

\footnotetext{
a) Submitted as part of the Proceedings of the 22nd Topical Conference on High-Temperature Plasma Diagnostics, San Diego, California, April, 2018.

b) Electronic mail: florian.penzel@ipp.mpg.de
}

interface definition and as a general challenge the high integration needs. Additionally, the applicability of current day bolometer systems which can be built upon is very limited, thus many technologies and subsystems must be re-developed and certified to be suitable for the ITER environment. This paper will describe the currently proposed system level design (SLD) of the bolometer port plug camera and its subcomponents.

\section{SYSTEM LEVEL DESIGN OF THE PORT PLUG CAMERAS AND ITS SUBCOMPONENTS}

Figure 1 shows a CAD drawing of the 5 bolometer port plug cameras (red) projected from two sectors (Sector 01 and 17) into one poloidal plane. The cameras are shown embedded into their port plug structure, the routing of the mineral insulated cables (MIC) is indicated exemplary for the horizontal Equatorial Port Plug (EPP) camera. The LOS and their viewing cones are indicated in yellow, though not all of them are shown. Three cameras are contained in the EPP 01 and two cameras in each of the two Upper Port Plugs (UPP) 17 and 01 , respectively. The cameras contained in UPP17 and UPP01 are in principle equally designed but provide redundancy and enhanced lateral resolution by having interlaced LOS. 70 LOS are distributed into a 10 LOS pinhole camera viewing the upper edge plasma and a 60 LOS collimator/pinhole camera viewing the core plasma from top to bottom and the divertor. The bottom and top EPP cameras located in the Diagnostic Shield Module (DSM) 3 of the port and are viewing the low field side edge above the midplane and the divertor region, respectively. The central EPP camera is viewing the core 
plasma from the horizontal plane and is located in the DSM 2. The SLD will be explained on the basis of the UPP cameras, exploded views can be seen in figure 2 and 3. SLD of the EPP cameras is based on the same SLD principles and components as for the UPP cameras, therefore a detailed presentation of the EPP cameras is omitted in this publication.

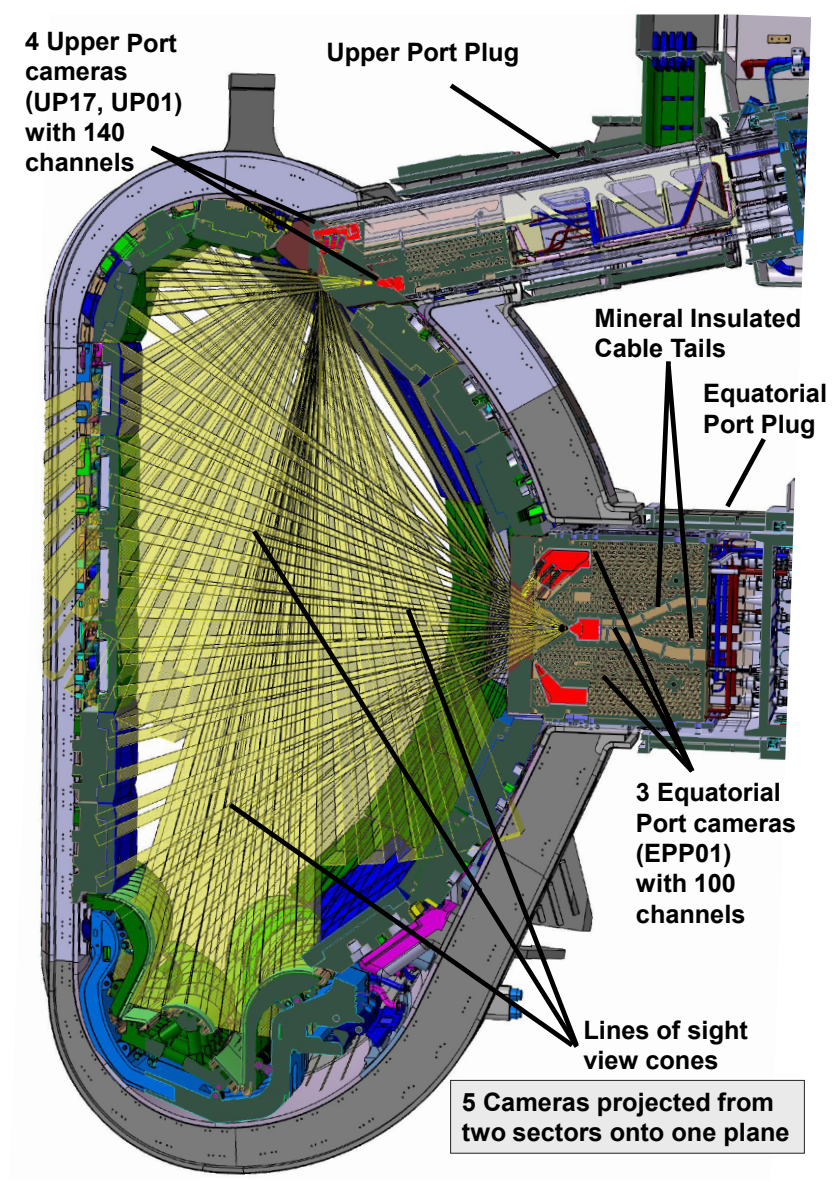

FIG. 1. ITER Bolometer Port Plug Cameras (red) and their LOS (yellow). LOS are shown only partly.

All port cameras are foreseen to be attached with M8 captive bolts to the interfacing DSM in the port plug. The connection is limited to the top and bottom side of the camera, the positions of the bolts are indicated in both camera drawings. The diagnostic needs direct viewing access to the plasma, therefore significant heating loads are impacting the structure which implies the use of a base materials with a high thermal conductivity such as TZM or CuCrZr combined with a high thermal contact conductance interface to the DSM.

For the internal camera design, four main items have to be considered: The required sensor positions (poloidally and radially) in order to view the desired observation area, the limited space envelope within the DSM, the required lateral resolution to achieve the target performance and the space needed for the camera components defining the LOS geometry. In order to determine the optimal camera geometry and e.g. questions regarding the design of multi-aperture collimators, an optimisation as described ${ }^{4}$ is performed.

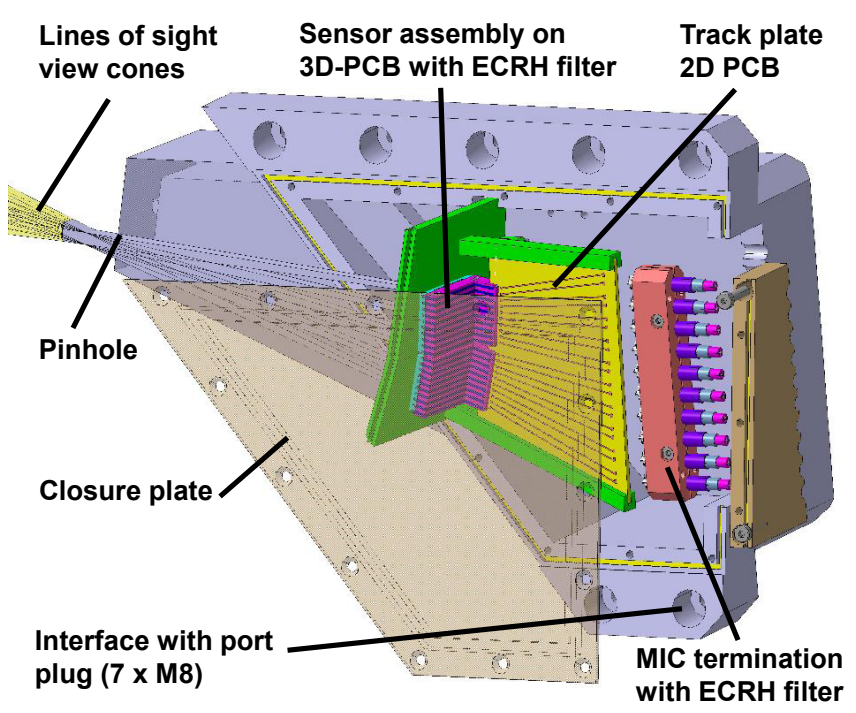

FIG. 2. UPP pinhole camera with 10 LOS - exploded view

All ITER in-vessel diagnostics must use Mineral Insulated Cables (MIC) for the electrical connection as they are the only cables qualified for the in-vessel nuclear and vacuum environment. The quadcore MIC foreseen for the bolometers have an outer diameter of $\varnothing_{M I C}=4 \mathrm{~mm}$ and the termination has a diameter of $\varnothing_{M I C-T}=8.6 \mathrm{~mm}$. As metal resistor bolometers use a Wheatstone bridge circuit to measure the incident radiation, each bolometer channel requires the connection with a single MIC: 60 LOS require 60 quadcore MIC.

The state-of-the art bolometer sensor design (as used on W7-X, ASDEX Upgrade, EAST, MAST-U, LHD...) features a four channel detector on a single chip. For ITER, it is envisaged to integrate five channels within one chip resulting in a chip size of about $23 \mathrm{~mm} * 25 \mathrm{~mm}$. However, it is not yet decided if those will consist of five individual channels or five channels on one chip. The design of the sensor holder presented here, is compatible with both options. For the integration, the chip is clamped in a ceramic holder having a front plate with cut outs for each absorber and a back plate, to which macroscopic electrical connection are to be made by a bonding process. The design of the sensor holder assembly can be seen in figure 4 Step 2, the dimensions of a four channel sensor prototype are shown in Step 1. The distance between the bond pads of one bolometer channel is $d_{\text {pads }}=2.5 \mathrm{~mm}$. An electrical connection is needed from the MIC entering the camera from one side and the sensors concentrated on the other side of the cameras. To solve the issue of these size relations and the three dimensional (3D) electrical routing requirements within the constrained space, it is envisaged to use ceramic printed circuit boards (PCB) based on AIN or Al2O3. 

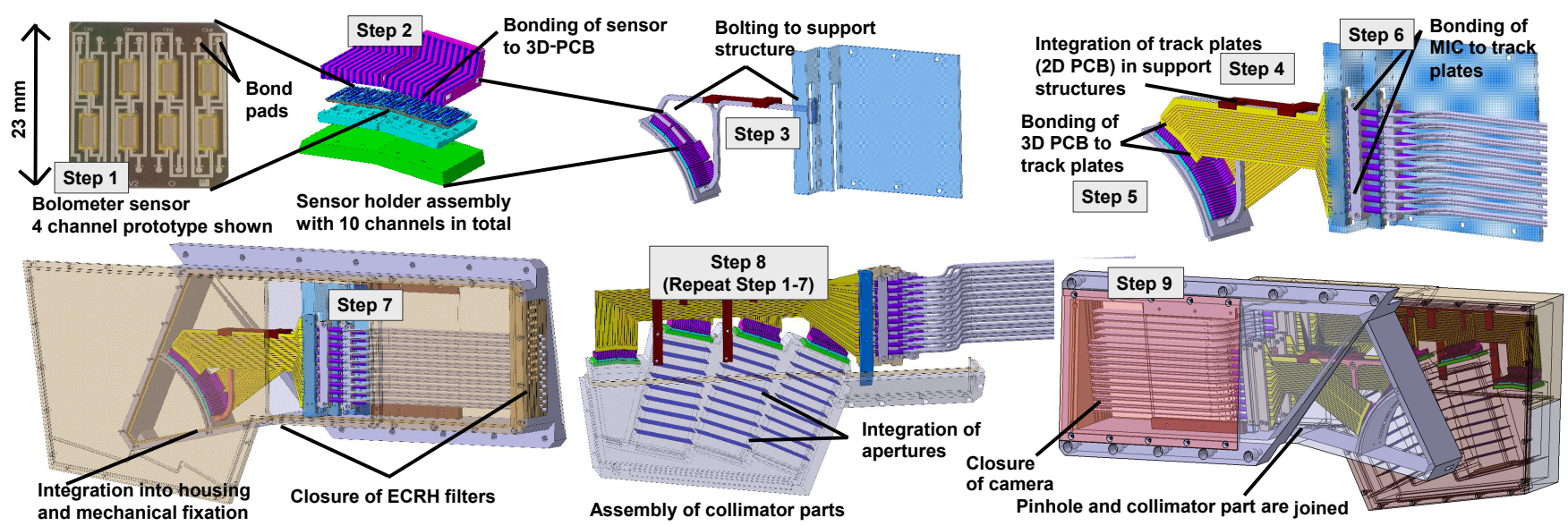

FIG. 4. Assembly concept of the UPPP 60 LOS collimator/pinhole camera.

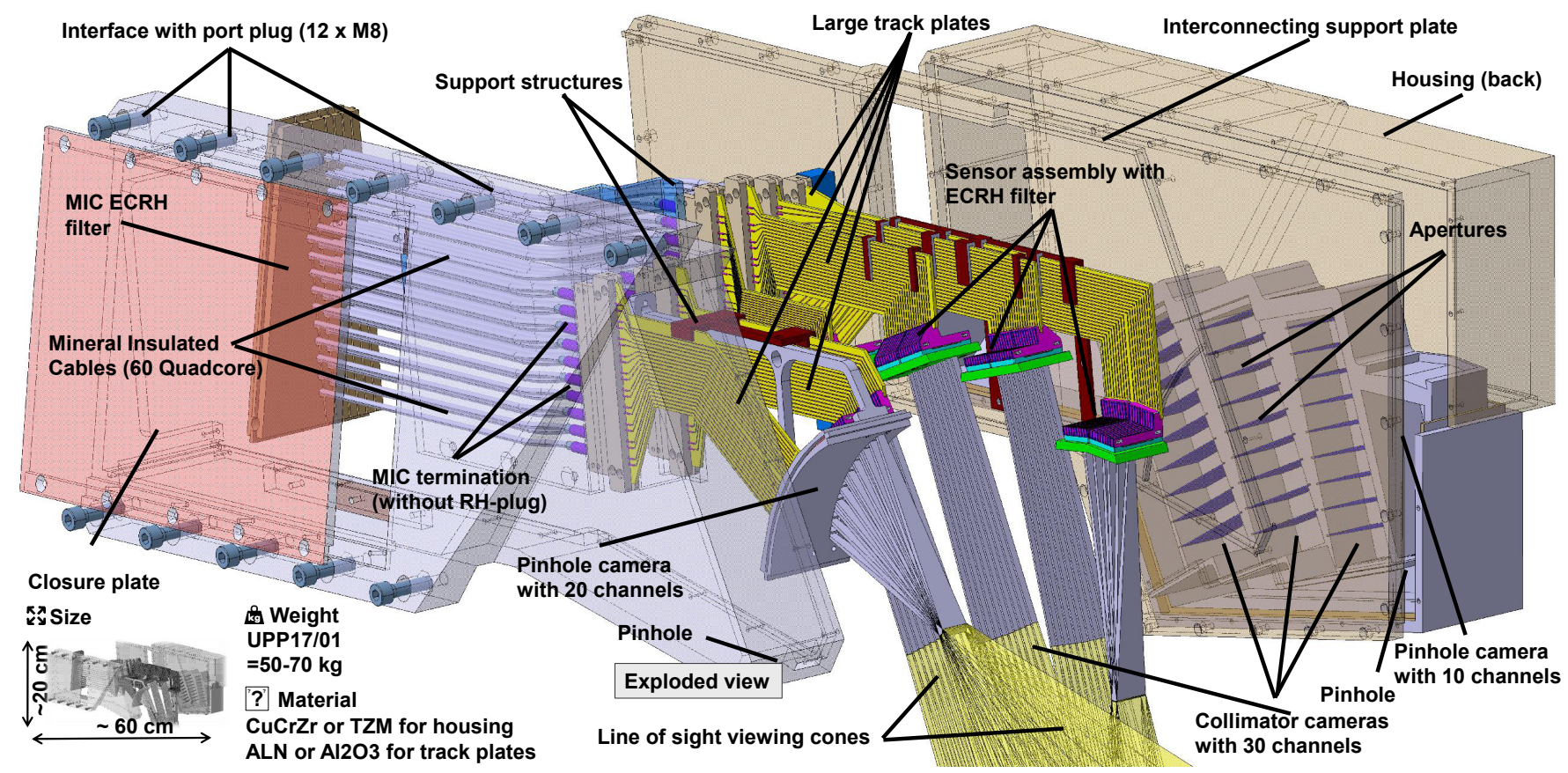

FIG. 3. ITER UPP bolomoter pinhole/collimator camera with 60 LOS - exploded view.

The electrical connection between those parts is then realized with a wire bonding process. Different methods are currently under investigation (ultra-sonic wedge bonding, capacitive discharge bonding, laser bonding). The bolometer sensor is bonded to the sensor holder back plate which constitutes of a 3D PCB. This sensor assembly connects to a flat $2 \mathrm{D}$ track plate which then routes and spreads out the MIC. The connection to the macroscopic MIC termination pin from the $2 \mathrm{D}$ track plate is also realized by a bond process. All sensor assemblies and track plates are held by different metallic support structures fixing these parts relative to each other.
The whole camera housing needs to be sufficient tight for ECRH stray radiation $\left(10 \log _{10}\left(P_{E C R H} / P_{\text {rad }}\right) \geq\right.$ $45 \mathrm{db}$ for the surfaces with the highest loads) in order to reduce the residual ECRH induced signal significantly below $P_{\text {rad }}{ }^{5}$. This requires the implementation of ECRH filter grids $\left(\varnothing_{\text {hole }}<\lambda / 2\right)$ into each bolometer channel which in this design is forseen to be implemented into the sensor assembly. To prevent microwave leakage through the MIC interface and the housing joints, a labyrinthlike dogleg structure with copper based shielding meshes is foreseen. It is required to shield the whole interior from microwave penetration as the ceramic based PCBs 
would otherwise be heated due to their good microwave absorption properties. In the end, the assembled 60 LOS UPP camera will have a weight of approximately $60 \mathrm{~kg}$ with dimensions of about $60 \mathrm{~cm} \times 50 \mathrm{~cm} \times 20 \mathrm{~cm}$.

\section{ASSEMBLY CONCEPT EXEMPLARY FOR THE UPPER PORT COLLIMATOR/PINHOLE CAMERA}

The assembly process is explained using the example of the 60 LOS UPP camera. For this camera, the assembly is divided into nine steps and is shown in figure 4. The first step is to integrate the bolometer sensor into the sensor assembly by clamping it between the front and back plate. The front plate has cut-outs for the detectors to face the plasma and the back plate access holes to allow wire bonding (Step 2) to the 3D PCB. For the pinhole cameras, the ECRH filter grid is integrated into an additional front plate (shown in green). Two sensor holders and the ECRH filter grids are then bolted together and attached to a support structure (Step 3). It is additionally kept in place by an support arm (shown in light blue). Next (Step 4), the first large track plate (shown in yellow) can be fixed to the support structure. Wire bonding of the 3D PCB to the track plate can now be performed on both sides. One layer of 10 MIC is inserted and bolted onto the support structure followed by wire bonding from the MIC to the track plates on both sides. Then, steps 4-6 are repeated to attach the next layer of MIC. The assembled structure forms now (Step 6) a 10 LOS pinhole camera subassembly.

The subassembly is integrated into the camera housing by additional bolting and adding the ECRH filters at the cable inlet. Step 8, which is the assembly of the collimator parts of the camera matches in principle steps 1-7 with the additional integration of the collimator apertures which can be done at any point of the process. The crucial point of the assembly is the gradual - layer by layer - insertion of the large track plates with the sensor holder PCBs in order to provide unobstructed bond access. A total of 720 bonds (3 bonds per channel x 60 channels $\mathrm{x} 4$ wires per channel) have to be performed on that camera.

\section{ARCHITECTURE OPTION COMPARISON ENABLING REMOTE HANDLING (RH) REPLACEMENT}

As already stated in section I, the bolometer diagnostic is classified RH class III as it does not require scheduled remote maintenance but is still likely to require unscheduled and very infrequent remote maintenance in case it would prevent ITER operation. Thus, two different architecture options (AO) allowing the complete replacement of the PP cameras have been investigated. Figure 6 illustrates AO 1 and 2.

For AO 1, the camera, the cable tail and the port feedthrough are treated as one component which is ex-

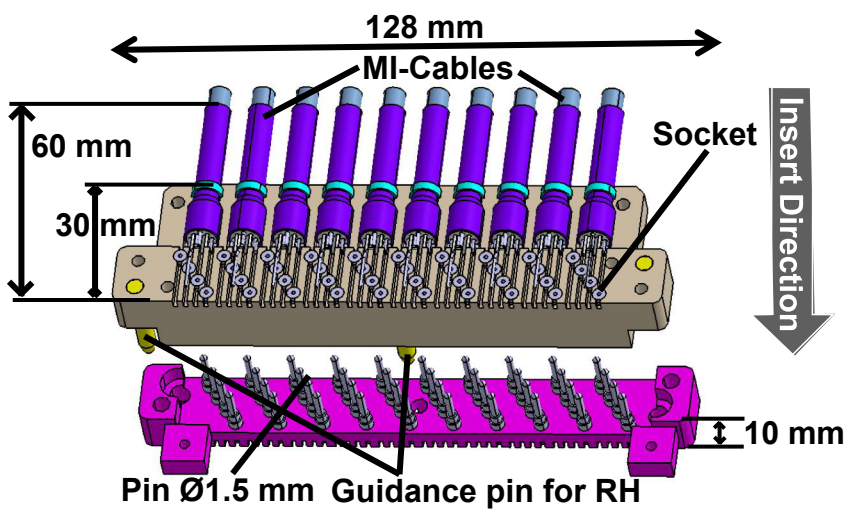

FIG. 5. Remote handling connector for option 2.

changed as a whole. The process is exemplarily shown using the example of the horizontal EPP camera. Water cooling pipes would be cut and weld with an auxiliary manipulator. For $\mathrm{AO} 2$, a $\mathrm{RH}$ connector is foreseen to be implemented in the middle of the camera, here shown exemplary for the 60 LOS UPP camera. The disconnection line is indicated with a red line. During $\mathrm{RH}$ the part containing the MIC stays in the DSM and only the camera containing the PCBs, the sensor and the parts defining the camera optics are removed. A connector design using pins and sockets (design adapted from STAUBLI connectors) has been developed and is shown in detail in figure 5. A market research and an analysis of already existing RH connectors in ITER has been performed, however none of those connectors did meet the requirements of the bolometer connectors, in particular due to the high amount of connections needed within the restricted space. The proposed connector allows to dis/connect 10 quadcore MIC within dimensions of about $130 \mathrm{~mm}($ width $) \times 25 \mathrm{~mm}($ height $) \times 30 \mathrm{~mm}($ length $)$ and features three RH guiding pins and 40 pins/sockets contained in a ceramic carrier. Connection pressure and locking is realized by captive bolts operating laterally towards the insert direction. A detailed review of both AO's has been performed estimating the impact on expected diagnostic performance, reliability, manufacturing cost and development effort, impact on cost for replacement, impact on electrical and sensor performance and general feasibility risks. AO 1 was selected. It is expected that a fully bonded electrical connection from sensor to feedthrough will provide superior reliability and performance. Also, the RH compatibility of the proposed connector has a significant higher risk of failure during replacement and the long-term reliability of such a connector is uncertain. Furthermore, A02 has been estimated to be significant more expensive due to the development effort for an new in-vessel compatible RH connector. 


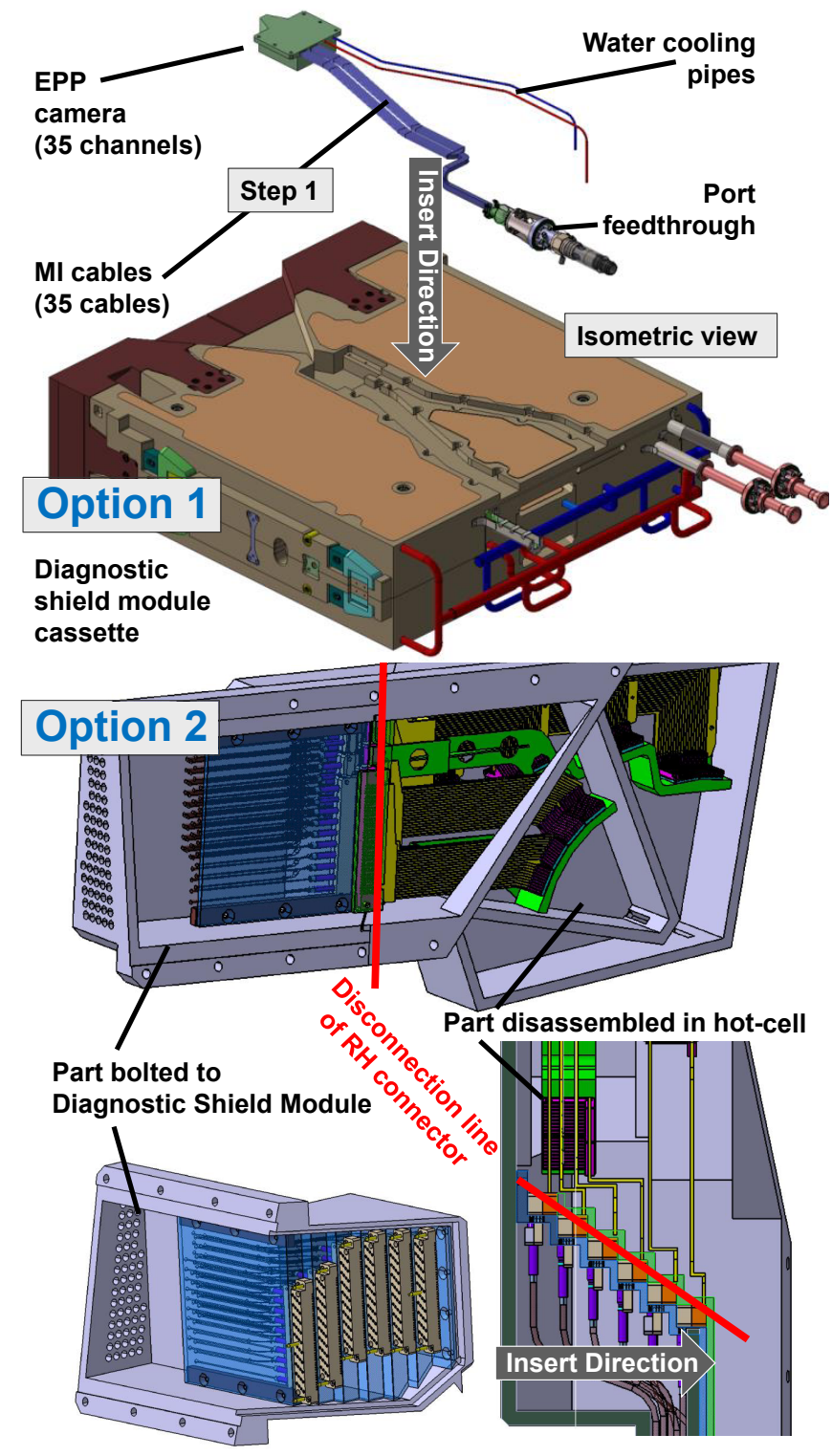

FIG. 6. Two options for remote handling replacement.

\section{CONCLUSIONS AND NEXT STEPS}

All functions that play an important role in determining the SLD of the ITER Bolometer Port Plug cameras have been identified and solutions proposed. Currently no potential show stoppers for the realization of the bolometer port plug cameras have been identified. The presented AOs are also compatible with minor changes, e.g. optimization of LOS distribution. However, there are a number of areas of concern which will have to be investigated closer: 1) The thermal management of the camera needs to be optimized and investigated, in particular to establish for which port cameras internal cooling is required. Current calculations indicate that cooling may be required for several PP cameras. Space requirements for cooling pipes have already been accounted for, however a detailed design will have to be developed once the decision has been taken. 2) EM loads during Vertical Disruption Events will cause significant accelerations (up to $26 \mathrm{~g}$ ) and vibrations on the bolometer subcomponents and there is the uncertainty of the internal structural integrity. In particular, the interface attachment concept of the ceramics supported by the metal support are under further investigation including finite element analysis. 3) The assembly and electrical connection of the connection between the 3D PCB sensor holder and the 2D track plate and between the MIC and the 2D track plate has to be realized under very restricted space and should be further investigated. One approach could be to perform a mock-up assembly test to make sure that there is sufficient space available for the different currently proposed bonding processes. 4) Finally, the investigation of manufacturing cost optimisation and the reduction of assembly complexity is planned in order to reduce overall cost, effort and reduce risks causing delay in the schedule on the way to realize the final cameras.

\section{ACKNOWLEDGMENTS}

This work was partly supported by Fusion for Energy under the Grant F4E-FPA-384-SG03. The views expressed in this publication are the sole responsibility of the authors and do not necessarily reflect the views of Fusion for Energy and the ITER Organization. Neither Fusion for Energy nor any person acting on behalf of $\mathrm{Fu}-$ sion for Energy is responsible for the use, which might be made of the information in this publication. The authors greatly acknowledge the contribution by IDOM for the assessment of the $\mathrm{RH}$ architecture options.

${ }^{1}$ K. Mast, J. Vallet, C. Andelfinger, P. Betzler, H. Kraus, and G. Schramm, Review of Scientific Instruments 62, 744 (1991).

${ }^{2}$ H. Meister, L. Giannone, L. D. Horton, G. Raupp, W. Zeidner, G. Grunda, S. Kalvin, U. Fischer, A. Serikov, S. Stickel, and R. Reichle, Review of Scientific Instruments 79, 10F511 (2008).

${ }^{3}$ F. Penzel, H. Meister, L. Giannone, M. Kannamüller, J. Koll, T. Trautmann, and A. W. Koch, Fusion Engineering and Design, Proceedings of the 27th Symposium of Fusion Technology (SOFT27) 88, 1267 (2013).

${ }^{4}$ H. Meister and S. Kalvin, Fusion Engineering and Design 89, 3039 (2014).

${ }^{5}$ H. Meister, W. Kasparek, D. Zhang, M. Hirsch, J. Koll, and A. Zeitler, Fusion Engineering and Design 96-97, 861 (2015), proceedings of the 28th Symposium On Fusion Technology (SOFT28). 